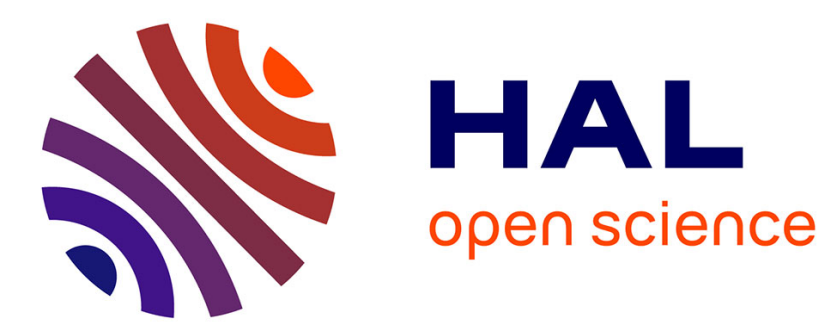

\title{
Filtration of Pitch-Class Sets Complexes
}

\author{
Louis Bigo, Moreno Andreatta
}

\section{To cite this version:}

Louis Bigo, Moreno Andreatta. Filtration of Pitch-Class Sets Complexes. 7th International Conference, MCM 2019, Jun 2019, Madrid, Spain. pp.213-226, 10.1007/978-3-030-21392-3_17 . hal02153236

\section{HAL Id: hal-02153236 \\ https://hal.science/hal-02153236}

Submitted on 2 Jul 2019

HAL is a multi-disciplinary open access archive for the deposit and dissemination of scientific research documents, whether they are published or not. The documents may come from teaching and research institutions in France or abroad, or from public or private research centers.
L'archive ouverte pluridisciplinaire HAL, est destinée au dépôt et à la diffusion de documents scientifiques de niveau recherche, publiés ou non, émanant des établissements d'enseignement et de recherche français ou étrangers, des laboratoires publics ou privés. 


\title{
Filtration of Pitch-class sets complexes
}

\author{
Louis Bigo $^{1[0000-0002-9865-2861]}$ and Moreno Andreatta ${ }^{20000-0002-7311-4741]}$ \\ 1 CRIStAL, UMR 9189, CNRS, Université de Lille, \\ Lille, France \\ louis.bigo@univ-lille.fr \\ 2 IRCAM/CNRS/Sorbonne Université, IRMA-GREAM, Université de Strasbourg, \\ Strasbourg, France \\ Moreno.Andreatta@ircam.fr, andreatta@math.unistra.fr
}

\begin{abstract}
A pitch-class set complex is a multidimensional object that spatially represents a collection of pitch-class sets and the intersections between them. If we consider the pitch classes within short time slices a piece can be divided into, we can evaluate for how long some combinations of pitch-classes sound simultaneously and then filter the piece according to the most relevant ones. This filtration process is performed by considering the superlevel sets of the function that computes the cumulative duration of pitch-class sets during the piece. Experiments show that musical sequences in the same style can exhibit similar sub-complexes in the filtration of their pitch-class set complexes. Filtered pitch-class set complexes also provide original informations on the use of the tonality and on the notion of centricity within a piece.
\end{abstract}

Keywords: Pitch-class sets · Harmonic similarity · Simplicial complexes · Pitch-class set complexes · Filtration · Persistent homology

\section{Introduction}

Pitch-class set theory, as a part of music set theory introduced by A. Forte [11], is largely used in musical analysis via a number of approaches. While mostly used for the study of atonal music, pitch-class set theory provides efficient tools for systematic study of any kind of music that can be represented with collections of pitch-classes. We propose in this paper a method to organize pitch-class sets occurring over a musical piece. Organizations resulting from different pieces can be compared to evaluate harmonic similarity and perform stylistic classification.

Harmonic similarity in symbolic musical data has been studied with various approaches including compression methods [1] and geometrical distances [8]. The idea of reducing a musical sequence by keeping only the structurally more important elements is a fundamental principle in Schenkerian analysis. Automatizing Schenkerian analysis however raises problems of multiple outputs and high computation times [15]. Rather, the reduction method proposed here is systematic and does not face any complexity issue. 

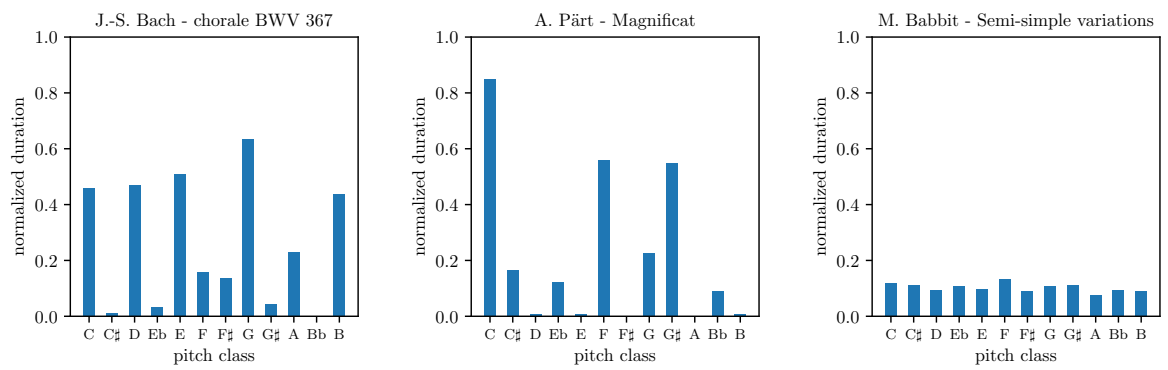

Fig. 1: Pitch-class distributions computed on different pieces.

In this work, pitch-class sets represent groups of notes that sound simultaneously. ${ }^{1}$ A musical piece is reduced to a sequence of successive slices, each slice being labelled by a pitch-class set. The cumulative duration of a pitch-class set over a musical piece is computed by summing the durations of the slices labelled by this pitch-class set. This value gives an indication of the importance of the pitch-class set over the whole piece. We use it as a filtration function to keep only predominant pitch-class sets.

Experiments show that two collections of predominant pitch-class sets, computed from distinct musical pieces, can be equivalent up to transposition. This equivalence indicates a similarity regarding the relative importance of pitchclasses and pitch-class sets in the two pieces and more generally regarding the way tonality is implemented in these pieces.

\subsection{Pitch-class Distributions and Centricity}

Pitch-class distributions enable to compare the cumulative duration of the 12 pitch-classes over a musical sequence. These distributions are computed by counting the number of occurrences of each pitch-class, generally weighted by their duration. Figure 1 displays pitch-class distributions of three musical pieces and illustrates different composition strategies in terms of pitch-class use: a tonal piece by J.-S. Bach, a tonal piece in the minimalist style by A. Pärt, and an atonal piece by M. Babbitt. Pitch-class cumulative durations are normalized by the duration of the piece.

Pitch-class distributions are used in a wide variety of applications, both in symbolic and audio music information retrieval. They are particularly used for chord recognition [14] and key detection, for which they can be compared to pre-defined pitch-class profiles [20].

Pitch-class distributions also constitute an efficient tool for studying centricity in a musical sequence. As discussed by D. Tymoczko [21], centricity relates

${ }^{1}$ Pitch-class sets in music set theory sometimes represent notes occurring close to each other but not necessarily simultaneously. 


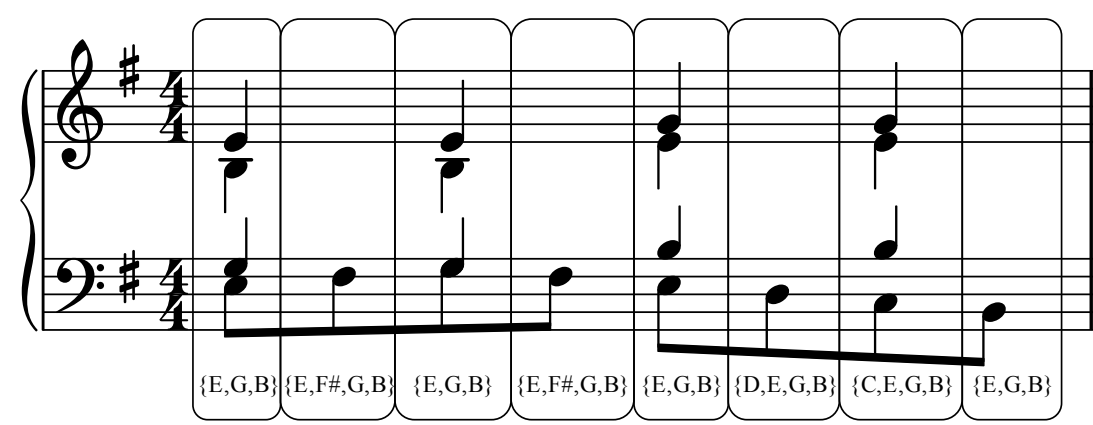

Fig. 2: Pitch-class set slicing of the first measure of chorale BWV 259 from J.-S. Bach. In this example, all slices have the duration of a eighth note.

to the idea that "a particular note is felt to be more prominent, important, or stable than the others." In this work, we extend the notion of centricity to sets of simultaneous pitch-classes. As the number of pitch-class sets appearing along a musical sequence can be large, we introduce the notion of filtration that enables to keep only the most relevant collections of pitch-class sets, based on their cumulative duration along the sequence. We use spatial representations of pitch-class sets to provide an intuitive understanding of these concepts.

\section{$1.2 \quad$ Spatial Representations}

Musical objects and their relations are frequently represented as spatial structures that give intuitive interpretations of music-theoretical principles. The (generalized) Tonnetz $[7,12,22]$ and voice-leading spaces [21] are examples of such representations and have been used in a wide variety of applications in music theory, analysis, and composition.

Geometrical simplicial complexes have been used to represent musical objects, in particular pitch-class sets $[18,6,5]$ and largely applied to stylistic and transformational analysis [4].

Recent studies into musical simplicial complexes and persistent homology [3, 13] and the increasing field of Topological Data Analysis have opened new perspectives for these approaches.

\section{Cumulative Duration of Pitch-Class Sets}

\subsection{Slicing Musical Sequences}

We represent musical sequences as ordered collections of consecutive pitch slices, each slice having a proper duration. Every time a new pitch starts or stops being played, the current slice ends and a new one begins. The process applies systematically for every note encountered within the score, including embellishments. This segmentation method, widely used in computational musicology, also goes 
under the name of pitch simultaneities or salami slicing [23]. It can be applied to any musical sequence of any genre as long as the sequence can be represented in the MIDI format. It can be straightforwardly generalized to represent pitch-class simultaneities by reducing every pitch set to its corresponding pitch-class set. ${ }^{2}$ A pitch-class set consists of any subset of $\mathbb{Z}_{12}$. The complete set of pitch-class sets corresponds to the set of subsets of $\mathbb{Z}_{12}$, called the power set of $\mathbb{Z}_{12}$ and notated $\mathcal{P}\left(\mathbb{Z}_{12}\right)$. Figure 2 illustrates this pitch-class set slicing process on the first measure of chorale BWV 259 from J.-S. Bach. More formally, a pitch-class slicing $\mathcal{S}$ is a sequence of $N$ pitch-class sets $A_{i}$ each accompanied by a duration $d_{i}$ :

$$
\mathcal{S}=\left[\left(A_{0}, d_{0}\right),\left(A_{1}, d_{1}\right), \ldots,\left(A_{N}, d_{N}\right)\right]
$$

where the durations $d_{i}$ sum up to the duration $D$ of the whole musical sequence.

\subsection{Cumulative Duration}

Let $\mathcal{S}$ be a pitch-class slicing and $X$ any arbitrary pitch-class set. The cumulative duration $\mathcal{D}_{\mathcal{S}}(X)$ corresponds to the sum of the durations of the slices of $\mathcal{S}$ whose pitch-class set includes $X$, normalized by the length of the entire pitch-class slicing $D$ :

$$
\mathcal{D}_{\mathcal{S}}(X)=\left[\sum_{\left\{\left(A_{i}, d_{i}\right) \in \mathcal{S} \mid X \subseteq A_{i}\right\}} d_{i}\right] / D
$$

The value $\mathcal{D}_{\mathcal{S}}(X)$ therefore indicates how much the pitch-class set $X$ appears in total along $\mathcal{S}$ and ranges between 0 (if the pitch-class set $X$ is absent from the sequence) and 1 (if the pitch-class set $X$ is played in each slice). The inclusion between pitch-class sets induces an ordering on their cumulative duration:

$$
\forall\left(X_{1}, X_{2}\right), X_{1} \subseteq X_{2} \Rightarrow \mathcal{D}_{\mathcal{S}}\left(X_{2}\right) \leq \mathcal{D}_{\mathcal{S}}\left(X_{1}\right)
$$

Figure 3 displays the 10 pitch-class sets having the highest cumulative durations within the 50 first slices of three musical excerpts. In order to facilitate the comparison between the examples, three sequences in the same key ( $\mathrm{G}$ major) have been selected.

\subsection{Ranking Pitch-Class Sets}

We call $\alpha_{i}^{n}$ the pitch-class set of size $n$ (or $n$-pitch-class set) having the $i$-th highest cumulative duration within a given musical sequence. For example, $\alpha_{1}^{1}$ is the most prevalent pitch-class and $\alpha_{2}^{3}$ is the second most prevalent 3-pitch-class set in the sequence. Therefore, the sequence $\alpha_{1}^{n}, \alpha_{2}^{n}, \ldots, \alpha_{m}^{n}$ gathers all $n$-pitchclass sets appearing in the sequence, sorted by decreasing cumulative duration, meaning that for every $i \in[0, m-1]$, we have $\mathcal{D}_{\mathcal{S}}\left(\alpha_{i}^{n}\right) \geq \mathcal{D}_{\mathcal{S}}\left(\alpha_{i+1}^{n}\right)$.

\footnotetext{
${ }^{2}$ In this work we restrain to twelve tone equal temperament in which pitch-classes are represented by integers modulo 12 .
} 

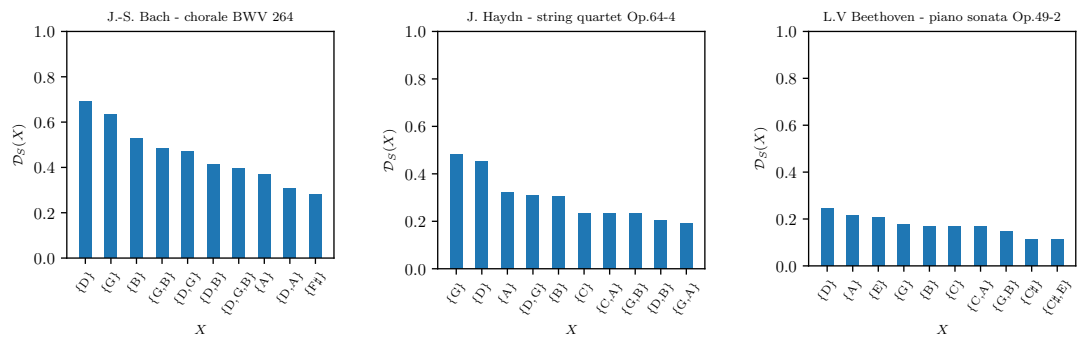

Fig. 3: 10 pitch-class sets having highest cumulative duration over the 50 first slices of 3 musical excerpts. The three excerpts are in the key of G Major.

\section{Pitch-Class Set Complexes}

\subsection{Representing Pitch-Class Sets}

We use labelled geometric simplices to represent pitch-class sets and labelled geometric simplicial complexes to represent collections of pitch-class sets. This approach goes back to Guerino Mazzola's Mathematical Music Theory $[17,18]$ which has been enriched recently to study aspects of generalized Tonnetze $[6,5]$.

A simplex of dimension $n$, or $n$-simplex, represents a pitch-class set of size $n+1$. In particular:

- a single pitch-class is represented by a 0 -simplex (a vertex),

- a 2-pitch-class set is represented by a 1-simplex (an edge),

- a 3-pitch-class set is represented by a 2 -simplex (a triangle),

- a 4-pitch-class set is represented by a 3 -simplex (a tetrahedron),

- a 5 -pitch-class set is represented by a 4 -simplex (a pentatope)

We say that a simplex is labelled by a pitch-class set and we denote by $\lambda(\sigma)$ the pitch-class set labelling the simplex $\sigma$. As in this work a simplex is systematically labelled by a unique pitch-class set, we will simplify the notation $\mathcal{D}_{\mathcal{S}}(\lambda(\sigma))$ by writing $\mathcal{D}_{\mathcal{S}}(\sigma)$ to designate the cumulative duration of the pitchclass set labelling the simplex $\sigma$.

The faces of a $n$-simplex $\sigma$ are the simplices incident to $\sigma$ whose dimension is lower than $n$. Simplices verify the closure condition which requires that a $n$-simplex is systematically bounded by $n+1$ faces of dimension $n-1$, and recursively. For instance, a 2-simplex (a triangle) is bounded by three 1-simplices (edges) which are each bounded by two 0-simplices (vertices). The faces of a simplex $\sigma$ represent subsets of the pitch-class set labelling $\sigma$. The closure condition results in the systematic representation of every subsets of pitch-classes included in a pitch-class set, which also fits with a perceptual and cognitive assumption. In fact, one could argue that during the listening process, a listener is hearing all possible subsets of notes included in a played chord. 


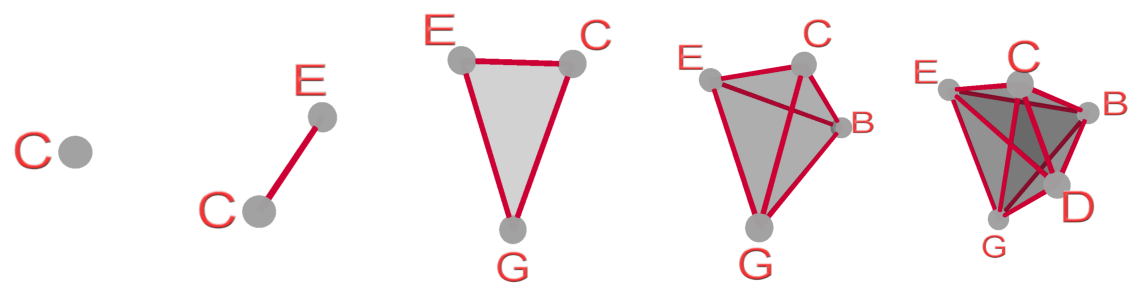

Fig. 4: Projection in $\mathbb{R}^{3}$ of $5 n$-simplices, respectively representing $(n+1)$-pitchclass sets, with $n=0,1,2,3$ and 4 .

Figure 4 displays simplices representing pitch-class sets of various size. The goal of these representations of pitch-class sets is to highlight inclusions between subsets via incidence relationships between simplices. The coordinates of the vertices in the figure do not matter and have been arbitrarily chosen to facilitate the visualization.

\subsection{Representing Collections of Pitch-Class Sets}

A pitch-class set slicing $\mathcal{S}$ can be represented by a pcs-complex $\mathcal{K}_{\mathcal{S}}$ which is obtained by:

1. representing every pitch-class set included in $\mathcal{S}$ by a simplex,

2. merging simplex faces that represent the same pitch-class set.

The merging step ensures that a given pitch-class set can label one simplex at most [5]. Figure 5 illustrates two pcs-complexes. The right pcs-complex gathers the pitch-class sets included in the pc-slicing of Figure 2. It consists in 33 simplices (tetrahedra) that share a common 2 -simplex (a triangular face) labelled by the pitch-class set $\{E, G, B\}$ (in dark gray in the figure).

\subsection{Filtration of Pcs-Complexes}

In this section, we introduce the idea of applying a filtration on a pcs-complex in order to keep only pitch-class sets that sound the most frequently over a musical piece. This process can be formalized by filtration functions.

A filtration function is a function $f: \mathcal{K} \rightarrow \mathbb{R}$, that associates a real number to any simplex of a simplicial complex $\mathcal{K}$ and that is non-increasing ${ }^{3}$ on increasing sequences of faces. This means that $f\left(\sigma_{1}\right) \geq f\left(\sigma_{2}\right)$ whenever $\sigma_{1}$ is a face of $\sigma_{2}$ in $\mathcal{K}$.

\footnotetext{
${ }^{3}$ Note that filtration functions can equally be defined as non-decreasing on increasing sequences of faces as long as $f$ is monotonic. In this paper we define filtration functions as non-increasing since this enables a more intuitive comprehension of the notion of filtering pitch-class sets over a musical piece depending on their cumulative duration as presented in section 4.
} 

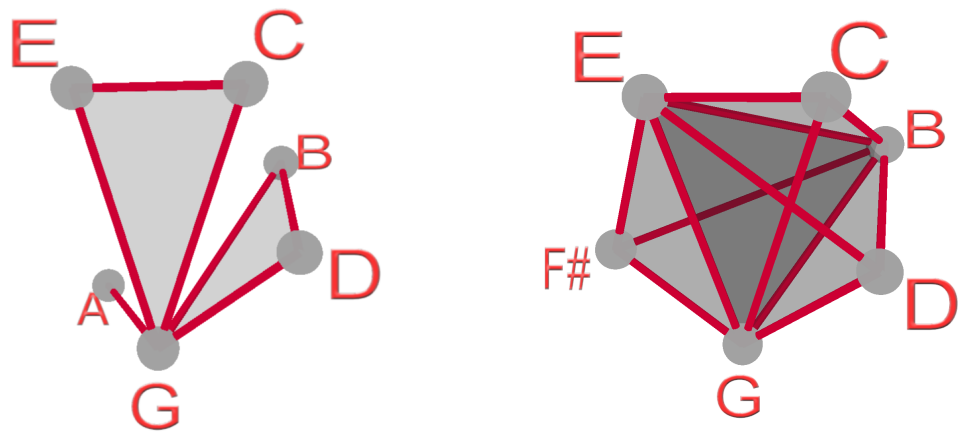

Fig. 5: On the left, a pcs-complex representing the collection of pitch-class sets $\{(C, E, G),(D, G, B),(G, A)\}$. On the right, the pcs-complex representing the collection of pitch-class sets included in the slicing illustrated on Figure 2.

Given a filtration function $f$ and a constant value $c \in \mathbb{R}$, we define the superlevel complex $\mathcal{L}_{c}^{+}(f)$ as the sub-complex of $\mathcal{K}$ that includes simplices for which $f$ attributes a value superior or equal to $c$ :

$$
\mathcal{L}_{c}^{+}(f)=\{\sigma \in \mathcal{K} \mid f(\sigma) \geq c\}
$$

The monotonicity of $f$ on increasing sequences of faces ensures that any superlevel complex will respect the closure condition on its simplices. The set of superlevel complexes produced by a filtration function can be ordered as a sequence of nested complexes, that starts with the empty complex and ends with the complete complex $\mathcal{K}$ :

$$
\emptyset=\mathcal{K}_{0} \subseteq \mathcal{K}_{1} \subseteq \cdots \subseteq \mathcal{K}_{n}=\mathcal{K}
$$

We may think of a filtration process as a description of how to construct $\mathcal{K}$ by adding chunks at a time [9]. ${ }^{4}$ As the simplices forming pcs-complexes are labelled by pitch-class sets, any function that systematically attributes a real value to a pitch-class set and that is non-increasing on sequences of increasingly inclusive pitch-class sets, can potentially be used as a filtration function on a pcscomplex. In Section 3.4 we will use the cumulative duration of pitch-class sets as a filtration function on the pcs-complex representing the slicing of a musical sequence.

\footnotetext{
${ }^{4}$ This may appear counter-intuitive as the notion of filtration is usually seen as a process that consists in removing elements as opposed to adding them. We will nevertheless keep this property to stick with the standard notation used in simplicial complex filtration [9].
} 

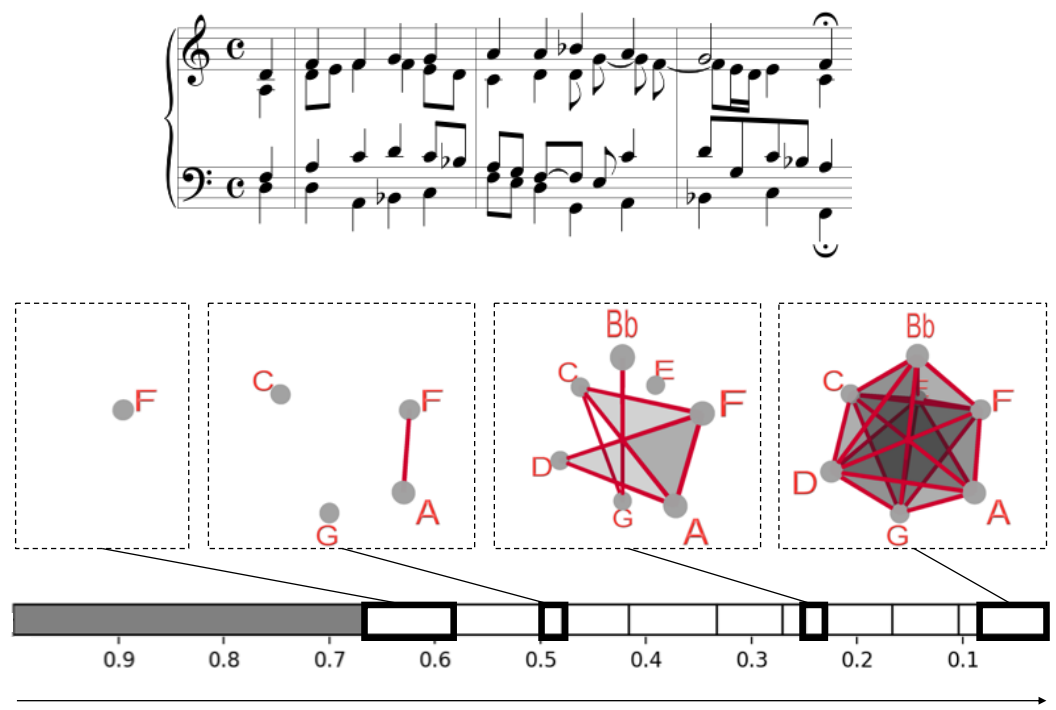

Fig. 6: The four superlevel complexes $\mathcal{L}_{0.66}^{+}, \mathcal{L}_{0.5}^{+}, \mathcal{L}_{0.25}^{+}$, and $\mathcal{L}_{0}^{+}$, induced by the filtration of the pcs-complex representation of the first phrase of J.-S. Bach chorale BWV 254. The bottom arrow represents the evolution of the filtration.

\subsection{A Filtration Based on Cumulative Durations}

When applied to pitch-class sets that label a pcs-complex, cumulative duration is a non increasing function on increasing sequences of faces. It can therefore be used as a filtration function on pcs-complexes.

As the cumulative duration $\mathcal{D}_{\mathcal{S}}$ is the only filtration function considered in this work, we take the liberty to simplify the superlevel complex notation from $\mathcal{L}_{c}^{+}\left(\mathcal{D}_{\mathcal{S}}\right)$ to $\mathcal{L}_{c}^{+}$:

$$
\mathcal{L}_{c}^{+}=\left\{\sigma \in \mathcal{K} \mid \mathcal{D}_{\mathcal{S}}(\sigma) \geq c\right\}
$$

Over the filtration of a pcs-complex based on cumulative duration, the first non-empty nested sub-complex $\mathcal{K}_{1}$ generally consists of one single 0 -simplex (a vertex) labelled by $\alpha_{1}^{1}$ which is the most prevalent pitch-class. ${ }^{5} \mathcal{K}_{2}$ generally consists of the 0 -simplex of $\mathcal{K}_{1}$ plus an additional 0 -simplex labelled by the second most prevalent pitch-class $\alpha_{2}^{1}$. $\mathcal{K}_{3}$ will generally either add a third 0simplex labelled by the third most prevalent pitch-class $\alpha_{3}^{1}$, or a 1-simplex (edge) labelled by the most prevalent 2-pitch-class set $\alpha_{1}^{2}$ which necessarily is $\left\{\alpha_{1}^{1}, \alpha_{2}^{1}\right\}$.

Figure 6 illustrates four superlevel complexes resulting from the filtration of the pcs-complex representing the first phrase of the chorale BWV 254 by J.-S.

\footnotetext{
${ }^{5}$ It can still happen, especially in short musical sequences, that the highest cumulative duration is equally attributed to two or more pitch classes. $\mathcal{K}_{1}$ therefore corresponds to a $n$-simplex with $n>0$.
} 


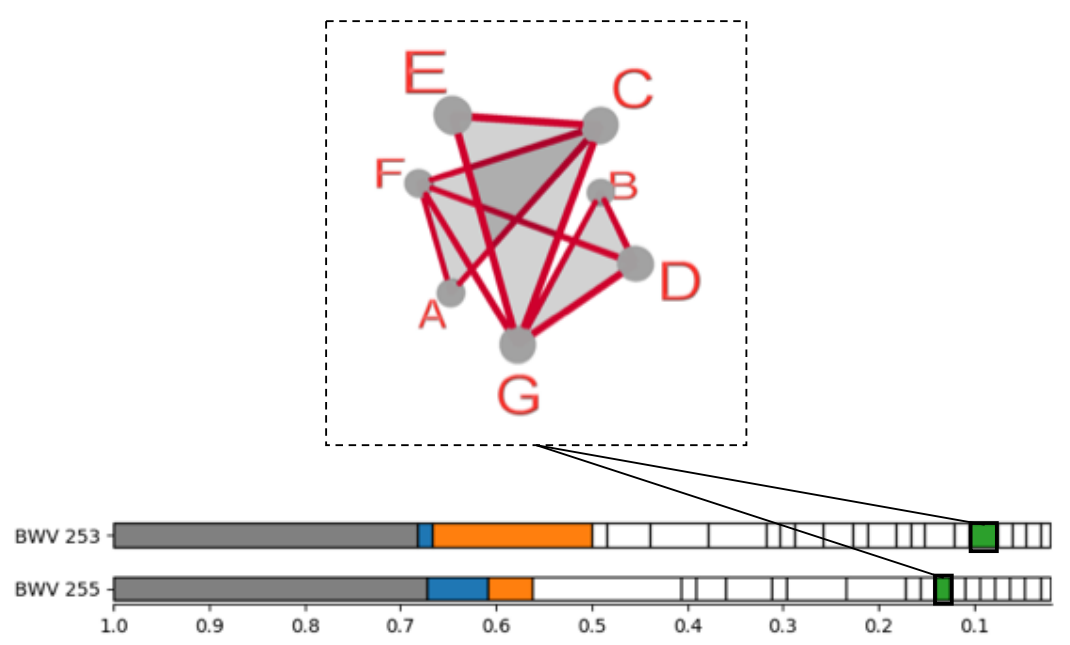

Fig. 7: Equivalence up to transposition of two superlevel complexes in the filtration of J.-S. Bach's chorales BWV 253 and BWV 255.

Bach. The horizontal bar represents the sequence of nested complexes resulting from the filtration. The filtration process goes from left to right. The first complex of the sequence is the empty complex $\mathcal{K}_{0}$ which appears in the leftmost region of the bar (gray). When the filtration level passes below 0.66, a 0-simplex $\left(\mathcal{K}_{1}\right)$ labelled by the most prevalent pitch-class $(F)$ appears. At the end of the filtration (right extremity of the bar) the complete collection of pitch-class sets that sound during this extract have appeared within the complex.

Filtering pitch-class sets that occur predominantly across a musical sequence provides a condensed representation of the pitch-class set material used in the sequence. In section 4 , we try to identify and interpret similarities between superlevel complexes resulting from the filtration of different musical pieces.

\section{Analyzing Filtrations}

\subsection{Transpositionally Equivalent Superlevel Complexes}

Figure 7 illustrates a superlevel complex that appears during the course of the filtration of both chorales BWV 253 and BWV 255. For the sake of clarity, the pcs-complex in the top of the figure has been transposed into a form that only exhibits pitch-class sets in the key of $\mathrm{C}$ major although the corresponding superlevel complexes in the filtration of BWV 253 and BWV 255 respectively gather pitch-class sets in the keys of A major and G major (see Table 1).

This common trait in the filtration of the two pcs-complexes can be interpreted in the following way: the collection of all pitch-class sets that are heard more than $10 \%$ of the time in the chorale BWV 253 is equivalent (up to a trans- 
position) to the collection of all pitch-class sets that are heard more than $14 \%$ of the time in the chorale BWV 255.

Intuitively, a similar superlevel complex illustrates the fact that a collection of pitch-class sets, that all occur predominantly in one sequence, is equivalent up to transposition to a collection of pitch-class sets that all occur predominantly in one other sequence. This property that shows a similarity between both harmonic contents, is not surprising for pieces in the same style. However, the size of the superlevel complexes and the level of the filtration at which they appear enable to measure in an original way this harmonic similarity. Similar superlevel complexes appearing at low level of the filtration are indeed especially striking as they demonstrate a strong similarity in the hierarchy linking the pitch-class sets appearing along the two sequences.

The pitch-class sets in Table 1 confirm a strong presence of tonality which is characteristic of the repertory of J.-S. Bach. Observing pitch-class sets that label superlevel complexes can also provide interesting information regarding the way the principle of tonality is implemented within the piece.

\begin{tabular}{|l|l|l|}
\cline { 2 - 3 } \multicolumn{1}{c|}{} & BWV 253 (T⿰亻⿻ & BWV 255 $\left(T_{7}\right)$ \\
\hline$\{0,4,7\}$ & $\{1,4,9\}$ & $\{2,7,11\}$ \\
$\{0,5,9\}$ & $\{2,6,9\}$ & $\{0,4,7\}$ \\
$\{2,7,11\}$ & $\{4,8,11\}$ & $\{2,6,9\}$ \\
$\{5,7\}$ & $\{2,4\}$ & $\{0,2\}$ \\
$\{2,5\}$ & $\{2,11\}$ & $\{0,9\}$ \\
\hline
\end{tabular}

Table 1: A collection of pitch-class sets in three forms equivalent up to transposition. The pitch-class sets in the first column label the complex illustrated on Figure 7. The pitch-class sets in the next two columns label superlevel complexes appearing respectively in filtration of pieces BWV 253 and BWV 255. Subsets of pitch-class sets are omitted to lighten notations.

Figure 8 illustrates the appearance of almost the same complex than the one in Figure $7,\{\{C, E, G\},\{C, F, A\},\{D, F, G, B\}\}$, in a low level of filtration of three movements of quartets by J. Haydn. The corresponding collection of pitchclass sets consists of three essential degrees of a major tonality $\left(I, I V\right.$ and $\left.V^{7}\right)$ and provides a sign of a strong tonal context within these pieces. Next appearing simplices in the filtration are (after the same transposition) $\{\{D, A\},\{D, F, A\}\}$ for Haydn Op. 33 No. 3-iii, $\{\{G, A\},\{C, G, A\}\}$ for Haydn Op. 17 No. 1-i and $\{\{G, A\},\{C, G, A\},\{C, B\},\{C, G, B\}\}$ for Haydn Op. 17 No. 6-i.

This experiment shows that looking at the superlevel complexes of the filtration of a musical piece can provide elements, in addition with pitch-class sets distributions, to study the prevalence of some underlying tonality. 


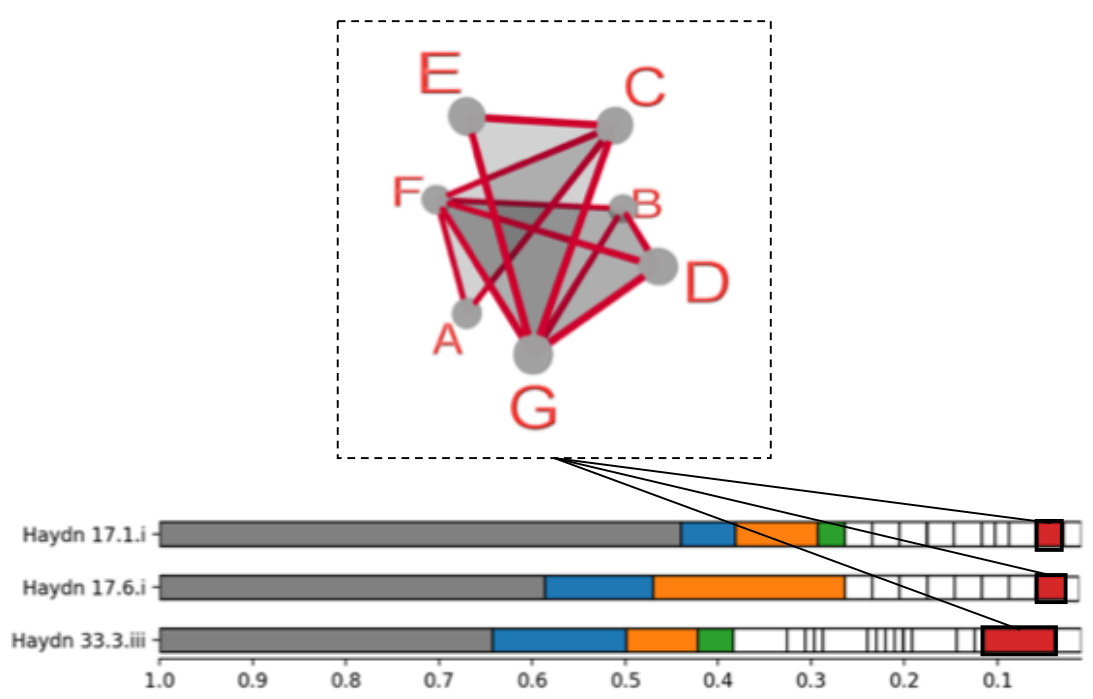

Fig. 8: Equivalence up to transposition of three superlevel complexes in the filtration of three movements of Haydn quartets.

\subsection{Discussion on Filtration Features}

Measuring the similarity between two filtrations is not straightforward and can be performed in different ways. We propose some filtration features that can be used as a distance measure to compare musical pieces.

The filtration of a pcs-complex $\mathcal{K}_{\mathcal{S}}$ can first be described by some features unrelated to the values of its pitch-class sets:

- the level from which the filtration provides a non-empty complex. This level corresponds to the cumulative duration of the most prevalent pitch-class set $\left(\mathcal{D}_{\mathcal{S}}\left(\alpha_{1}^{1}\right)\right)$, which is likely to be a single pitch-class.

- the maximum level at which the filtration of $\mathcal{K}_{\mathcal{S}}$ includes a 2 -simplex which represents a minor or major triad. This feature indicates how present is the most frequent triad.

A more extensive study could focus on step sizes between successive levels during a filtration process. For example, the difference of levels between the two first non-empty complexes gives an idea of how much more prevalent is the most prevalent pitch-class set over the remaining pitch-class sets. This measure closely relies to the notion of centricity which indicates how much a selected pitch-class (more generally a pitch-class set) is prevalent over all the others. Filtration of pcs-complexes therefore provides a collection of original tools to study centricity in musical sequences. 


\subsection{Persistence of Musical Properties}

Experiments described in Section 4.1 could be extended to track music similarity between two musical sequences for example by measuring the lowest filtration level exhibiting two superlevel complexes that are equivalent up to transposition.

The way tonality is implemented in a musical piece could also be studied by measuring the lowest level at which the filtration of its pcs-complex includes pitch-class sets that all belong to one usual tonality.

Large intervals between filtration levels, wherever they occur during the filtration, highlight a collection of pitch-class sets that is strongly more frequent than the rest and can also reach to original approaches for music analysis.

\subsection{Relations to Persistent Homology}

Looking at the "life duration" of a musical property during the course of a filtration process relates to the notion of "persistence" in persistent homology. In persistent homology, persistence relates to the life-cycle of a homology class over a filtration [10]. These homology classes are associated with topological features of the filtered complex, such as connected components, circular holes or cavities. Their persistence during the filtration process is represented by persistent diagrams which can be compared by using the bottleneck distance. Different experiments following this approach have proven to be promising for musical classification $[3,2,13]$. However, the difficulty to bring a musical interpretation of the life cycle of homotopy classes remains an obstacle to the full understanding of music-analytical techniques based on persistent homology.

Extending analysis approaches inspired by persistent homology would undoubtedly benefit from a number of tools and methods that have been elaborated in this field of mathematics and, more specifically, in the community of Topological Data Analysis (TDA).

\section{Code Implementation and Visualization}

The code developed for this research has been written in Python3 and is freely available online. ${ }^{6} 3 \mathrm{D}$ representations of pcs-complexes have been produced with a dedicated tool accessible through any internet browser. ${ }^{7}$ Musical sequences are uploaded in this tool as MIDI files. The browser displays a 3D representation of the pcs-complex gathering every set of pitch classes that happen to sound simultaneously at some point of the sequence. The analysis can be reduced to any time window within the sequence thanks to the double horizontal cursor. A vertical cursor enables the user to select a filtration level that determines the displayed simplices. The values taken by the cursor run from 0 to 1 to stick with the definition of the normalized cumulative duration of pitch-class sets.

\footnotetext{
${ }^{6}$ https://gitlab.com/lbigo/pitchsalami

${ }^{7}$ http://lbigo.gitlab.io/ChordComplexViz
} 


\section{Conclusion}

This work presents some experiments on filtration of pcs-complexes as a structural way to retrieve music information. We suggested some preliminary features to describe and compare filtrations. Future works include measuring the ability of these features to reveal musical similarities between different pieces, and more generally to provide some insights on the musical style of the represented piece. Future works also include a more systematic and in-depth study of the persistence of transposition relation between two filtered complexes, as the persistence of other musical relations such as inversion, diatonic transposition and, more generally, transformational operators.

Filtrations of pcs-complexes could also benefit from being reduced to selected temporal windows within a musical piece in order to exhibit changes of strategies in the use of pitch-class sets during the piece. Such approaches could undoubtedly benefit from multi-timescale visualization techniques that have been elaborated for various tasks including key detection [19] or set-class analysis [16].

Future experiments will also include the possibility to filter a pcs-complex reduced to a particular type of pitch-class set. For example, keeping only major and minor triads (and their subsets of pitch-classes) in a pitch-class slicing might more easily produce common superlevel complexes over the filtration of tonal musical sequences.

Acknowledgments. We would like to thank friends and colleagues for fruitful discussions and careful proofreading including Mattia Bergomi, Paul Ladyman, members of the spatial computing project and the Algomus team. We also thank Antoine Lafrance for his contribution on the online visualization application.

\section{References}

1. Ahonen, T.E., Lemström, K., Linkola, S.: Compression-based similarity measures in symbolic, polyphonic music. In: ISMIR. pp. 91-96 (2011)

2. Bergomi, M.G., Baratè, A., Di Fabio, B.: Towards a topological fingerprint of music. In: International Workshop on Computational Topology in Image Context. pp. 88-100. Springer (2016)

3. Bergomi, M.G.: Dynamical and topological tools for (modern) music analysis. Ph.D. thesis, Université Pierre et Marie Curie-Paris VI (2015)

4. Bigo, L., Andreatta, M.: Topological structures in computer-aided music analysis. In: Computational Music Analysis, pp. 57-80. Springer (2016)

5. Bigo, L., Andreatta, M., Giavitto, J.L., Michel, O., Spicher, A.: Computation and visualization of musical structures in chord-based simplicial complexes. In: International Conference on Mathematics and Computation in Music. pp. 38-51. Springer (2013)

6. Catanzaro, M.J.: Generalized tonnetze. Journal of Mathematics and Music 5(2), 117-139 (2011)

7. Cohn, R.: Neo-riemannian operations, parsimonious trichords, and their" tonnetz" representations. Journal of Music Theory 41(1), 1-66 (1997) 
8. De Haas, W.B., Wiering, F., Veltkamp, R.C.: A geometrical distance measure for determining the similarity of musical harmony. International Journal of Multimedia Information Retrieval 2(3), 189-202 (2013)

9. Edelsbrunner, H.: Geometry and topology for mesh generation, vol. 7. Cambridge University Press (2001)

10. Edelsbrunner, H., Harer, J.: Computational topology: an introduction. American Mathematical Soc. (2010)

11. Forte, A.: The structure of atonal music, vol. 304. Yale University Press (1973)

12. Gollin, E.: Some aspects of three-dimensional" tonnetze". Journal of Music Theory pp. 195-206 (1998)

13. Lascabettes, P.: Homologie Persistante Appliquée à le reconnaissance de genres musicaux. Master's thesis, École Normale Suprieure Paris-Saclay/Irma (2018)

14. Lee, K.: Automatic chord recognition from audio using enhanced pitch class profile. In: ICMC (2006)

15. Marsden, A.: Schenkerian analysis by computer: A proof of concept. Journal of New Music Research 39(3), 269-289 (2010)

16. Martorell, A., Gómez, E.: Contextual set-class analysis. In: Computational Music Analysis, pp. 81-110. Springer (2016)

17. Mazzola, G.: Geometrie der Töne: Elemente der Mathematischen Musiktheorie. Birkhäuser (1990)

18. Mazzola, G.: The topos of music: geometric logic of concepts, theory, and performance. Birkhäuser (2012)

19. Sapp, C.S.: Harmonic visualizations of tonal music. In: ICMC. vol. 1, pp. 419-422. Citeseer (2001)

20. Temperley, D., Marvin, E.W.: Pitch-class distribution and the identification of key. Music Perception: An Interdisciplinary Journal 25(3), 193-212 (2008)

21. Tymoczko, D.: A geometry of music: Harmony and counterpoint in the extended common practice. Oxford University Press (2010)

22. Tymoczko, D.: The generalized tonnetz. Journal of Music Theory pp. 1-52 (2012)

23. White, C.W., Quinn, I.: The yale-classical archives corpus. Empirical Musicology Review 11(1) (2016) 